\title{
Subcellular Localization of Bilirubin in Rat Brain after In Vivo i.v. Administration of $\left[{ }^{3} \mathrm{H}\right]$ Bilirubin
}

\author{
THOR WILLY RUUD HANSEN, STEVEN TOMMARELLO, AND JEFFREY W. ALLEN \\ Department of Anesthesiology and Critical Care Medicine, Children's Hospital, University of Pittsburgh, \\ Pittsburgh, Pennsylvania, U.S.A. [T.W.R.H., S.T., J.W.A.]; and Section on Neonatology, Department of \\ Pediatrics, Rikshospitalet, University of Oslo, Oslo, Norway [T.W.R.H.]
}

\begin{abstract}
ABST
Bilirubin appears to be toxic in vitro to several cellular
functions localized to different subcellular compartments. It
would therefore be useful to know what concentrations of bili-
rubin might be found in cell organelles in vivo. Rats were
anesthetized and allocated to one of three groups: control, hy-
percarbia, and hyperosmolality. Each rat received a 5-min bolus
dose of bilirubin 50 mg/kg i.v. (containing approximately 200
$\mu C i$ [ ${ }^{3}$ H]bilirubin). Rats were killed 10 or 30 min after the start
of the bilirubin infusion. Each brain was homogenized, and
subcellular fractions were isolated by high-speed gradient cen-
trifugation in sucrose media. The gradients were separated into
aliquots of 2 mL, and the protein content was determined in each
aliquot. Radioactivity was determined by scintillation counting,
and the content of bilirubin per milligram of protein was calcu-
lated. Statistical comparisons were performed with Kruskal-
Wallis nonparametric ANOVA. There were highly significant
differences in bilirubin content per milligram of protein among
subcellular compartments in all groups and at both time points. In
\end{abstract}
all groups there were relatively high concentrations of bilirubin in the myelin fraction, an interesting observation in light of the theory that membranes are the primary target of bilirubin toxicity. The very high concentration of bilirubin relative to protein in cytoplasm, ribosomes, and mitochondria in the hyperosmolar group are also notable in light of data from hyperbilirubinemic animals in which changes in electrophysiology or energy metabolism only appeared after hyperosmolar opening of the bloodbrain barrier. The present data may be useful in planning in vitro studies of bilirubin toxicity in cell organelles. (Pediatr Res 49: 203-207, 2001)
Abbreviations:
P1, P2, P3, P4, first, second, third, and fourth pellets from sequential density centrifugations
S1, S2, S3, S4, first, second, third, and fourth supernatants from sequential density centrifugations

Neonatal jaundice is a common and, in most infants, normal physiologic event, which has been discussed in the medical literature for at least a quarter of a millennium (1). Some jaundiced infants exhibit signs of neurotoxicity, which appears to be transitory in the great majority. Exceptionally, bilirubin toxicity may result in death in the newborn period or in survival with severe neurologic sequelae (kernicterus). During the past $50 \mathrm{y}$ considerable research effort has been directed toward the interaction between bilirubin and the brain, as well as the mechanism (or mechanisms) of neurotoxicity.

Much has been learned about how bilirubin enters into and is cleared from the brain, but the process that leads to deposition of bilirubin in basal ganglia and other brain regions to create the pattern known as kernicterus (jaundice of the nuclei) is as yet not understood. Also, none of the theories concerning

Received June 14, 2000; accepted September 7, 2000.

Correspondence and reprint requests: Thor Willy Ruud Hansen, M.D., Ph.D., Section on Neonatology, Department of Pediatrics, Rikshospitalet, N-0027 Oslo, Norway.

Supported by grants from Children's Hospital of Pittsburgh and the University Anesthesiology and Critical Care Medicine Foundation, Pittsburgh, PA, U.S.A. the basic neurobiological mechanism of bilirubin neurotoxicity have been conclusively proven.

A number of methodological problems have bedeviled bilirubin researchers and impeded progress. One of the more vexing is the near insolubility of bilirubin in aqueous media at physiologic $\mathrm{pH}$, making in vitro experiments difficult to perform without the presence of solubilizers or stabilizers, which may themselves perturb the reaction or process being studied. Another is the difficulty in knowing what constitutes a physiologically relevant bilirubin concentration for the particular process under investigation.

The mechanism (or mechanisms) by which bilirubin exerts its toxic effects on brain tissue is unknown, although a number of theories have at least some experimental support. One of the classic theories suggests that bilirubin uncouples oxidative phosphorylation (1-3). For this to happen bilirubin must enter the mitochondria of neurons in relevant concentrations. Perturbation of cell membrane function is another mechanism that has some experimental support (4-10). Yet other studies have shown effects of bilirubin on protein synthesis, neurotransmit- 
ter synthesis, DNA synthesis, and carbohydrate metabolism (11). Protein and peptide phosphorylation, an important regulatory mechanism in neurons, has also been shown to be inhibited by bilirubin (12-16). For the most part these effects have been demonstrated in vitro. However, before these effects can be said to represent possible mechanisms of bilirubin neurotoxicity, it must be shown that bilirubin enters the respective subcellular compartments in vivo.

Although information is available regarding bilirubin concentrations in whole brain and brain regions during induced hyperbilirubinemia in experimental animals (17-22), no studies are on record which track bilirubin entry into cells and subcellular compartments in vivo. The purpose herein was to study the subcellular localization of bilirubin in rat brain after in vivo administration of radioactively labeled bilirubin. Our hypothesis was that bilirubin would not distribute equally across subcellular compartments. The study was approved by the Animal Research and Care Committee, University of Pittsburgh, Pittsburgh, PA, U.S.A.

\section{METHODS}

Materials. Bilirubin, BSA, and urea were purchased from Sigma Chemical Co. (St. Louis, MO, U.S.A.). $\left[{ }^{3} \mathrm{H}\right]$ Bilirubin was synthesized by Moravek Biochemical, Inc. (Brea, CA, U.S.A.). The specific activity was $\geq 50 \mathrm{mCi} / \mathrm{mmol}$ and the purity was $\geq 90 \%$ as determined by HPLC by the manufacturer. Fifteen percent $\mathrm{CO}_{2}$ in air was obtained from a commercial supplier and was of medical grade. Ultima Gold scintillation fluid was from Packard Instrument Company (Meriden, CT, U.S.A.). Perfluorocarbon (Fluorinert FC-77) was from 3M Industrial Products Division (St. Paul, MN, U.S.A.). Other reagents, of analytical grade, were from standard commercial suppliers. Young, male Sprague Dawley rats $(100-200$ g) were obtained from Hilltop Lab Animals Inc. (Scottdale, PA, U.S.A.).

Preparation of the reagents. Unlabeled bilirubin was dissolved in $0.1 \mathrm{M} \mathrm{NaOH}$, stabilized with BSA (molar ratio bilirubin:albumin $=14$ ), and diluted with Krebs-Ringer buffer, $\mathrm{pH} 7.4$, to a concentration of $3 \mathrm{mg} / \mathrm{mL}$ (final $\mathrm{pH}$ approximately 8). Radioactive bilirubin was added to give each rat $200 \mu \mathrm{Ci}$ in a bilirubin bolus of $50 \mathrm{mg} / \mathrm{kg}$ i.v. over a period of $5 \mathrm{~min}$. Urea was dissolved in Krebs-Ringer buffer to a concentration of $8.3 \mathrm{M}$.

Preparation of the rat model. On the day of the experiment the rats were weighed, and then anesthetized with an s.c. injection of $1-2 \mathrm{~mL} / \mathrm{kg}$ of a mixture of fentanyl, droperidol, and midazolam $(0.025,1.25$, and $0.625 \mathrm{mg} / \mathrm{mL}$, respectively) (22). Anesthesia was maintained throughout the study by supplementary injections as needed. A venous catheter was placed in a dorsal foot vein for infusion, and a similar catheter was placed in the ventral tail artery. Rats were pretreated according to group allocation: control $(n=15)$, hypercarbia $(n$ $=14)$, or hyperosmolality $(n=14)$. Relative to control conditions, hypercarbia has been shown to increase brain bilirubin by increasing the short-term entry of bilirubin into brain (22). Hyperosmolality, on the other hand, increases the accumulation of bilirubin in brain over time by delaying its clearance from brain (22).

Rats in the hypercarbia group were placed in a large plastic bag approximately $12 \times 12$ inches through which $15 \% \mathrm{CO}_{2}$ in room air flowed at a rate of approximately $1 \mathrm{~L} / \mathrm{min}$, and remained in the bag throughout the experiment. Rats in the hyperosmolar group received an i.v. infusion over 3-4 min of $75 \mathrm{mmol} / \mathrm{kg}$ (45 mg/kg) urea (22). Control animals received an equivalent volume of lactated Ringer i.v.

Blood was drawn before sacrifice from the arterial catheter to monitor blood gases, and for measurements of serum total bilirubin and serum osmolality, and determination of specific activity of the $\left[{ }^{3} \mathrm{H}\right]$ bilirubin. Rats were killed with i.v. sodium pentobarbital $(100 \mathrm{mg} / \mathrm{kg})$ at either 10 or $30 \mathrm{~min}$ after the start of the bilirubin infusion. The chest was opened, the ascending aorta cannulated, the descending aorta clamped, the jugular veins transected, and the cerebral vasculature flushed in situ with $120 \mathrm{~mL}$ of ice-cold saline at a rate of $25 \mathrm{~mL} / \mathrm{min}$ (22). The brain was removed, carefully stripped of meningeal coverings and surface vessels, and stored in $0.32 \mathrm{M}$ sucrose on ice until further processing.

Preparation of subcellular fractions. Subcellular fractions were isolated by gradient centrifugation in sucrose media according to Gray and Whittaker (23) and Whittaker and Barker (24), as briefly described below. This is a well-tested method, which yields highly reproducible results. Brains were homogenized in a glass-Teflon homogenizer using $0.32 \mathrm{M}$ sucrose (volume in milliliters, $10 \times$ weight of brain in grams) using $2 \times 6$ strokes at $750 \mathrm{rpm}$ while cooling in ice sludge. The homogenate was centrifuged at $1000 \times g$ for $11 \mathrm{~min}(3500 \mathrm{rpm}$ in SA600 rotor in a Sorvall RC-5B Refrigerated Superspeed Centrifuge, Sorvall Instruments, Newtown, CT, U.S.A.). The supernatant (S1) was decanted, the pellet (P1) resuspended in $10 \mathrm{~mL} 0.32 \mathrm{M}$ sucrose, and recentrifuged as above. The supernatant from this latter centrifugation was added to S1. The pellet (P1) was resuspended to a volume of $2 \mathrm{~mL}$ in 0.32 $\mathrm{M}$ sucrose, and the protein content determined as described below.

The pooled S1 was pelleted by centrifuging at $17,000 \times g$ for $60 \mathrm{~min}(12,500 \mathrm{rpm}$ in the SA600 rotor). The supernatant (S2) from this procedure was set aside for subsequent isolation of microsomes and ribosomes, and the pellet (P2) was resuspended in $0.32 \mathrm{M}$ sucrose and carefully placed on top of a density gradient consisting of $2 \mathrm{~mL}$ of a high density solution (Fluorinert; "bottom pad"), 1.2 M (lower layer), and $0.8 \mathrm{M}$ (middle layer) sucrose. The gradient was then centrifuged at $53,000 \times g$ for $120 \mathrm{~min}(23,000 \mathrm{rpm}$ in a T- 865 rotor [DuPont/ Sorvall] using a Sorvall Ultracentrifuge OTD70B, Sorvall Instruments).

The gradient was drained off from the top in aliquots of 2 $\mathrm{mL}$ by carefully adding aliquots of $2 \mathrm{~mL}$ of Fluorinert to the bottom pad through a small-bore metal tube inserted through a rubber plug capping the tube. The gradient consisted of an upper layer $(0.32 \mathrm{M} / 0.8 \mathrm{M}$ interphase) containing myelin, a middle layer $(0.8 \mathrm{M} / 1.2 \mathrm{M}$ interphase) containing synaptosomes, and a lower layer containing mitochondria $(1.2 \mathrm{M} /$ bottom pad interphase). From each of the 2-mL aliquots $200 \mu \mathrm{L}$ was set aside to allow for determination of protein concentra- 
tion. The remainder of the aliquots was counted by scintillation as described below.

The S2 was centrifuged at $100,000 \times g$ for 60 min, sedimenting a $\mathrm{P} 3$ consisting of microsomes. The P3 was resuspended to $2 \mathrm{~mL}$ in $0.32 \mathrm{M}$ sucrose, analyzed, and counted by scintillation as described below. The supernatant S3 was decanted and centrifuged at $100,000 \times g$ for $120 \mathrm{~min}$. The P4, consisting of ribosomes, was resuspended to $2 \mathrm{~mL}$ in $0.32 \mathrm{M}$ sucrose, analyzed, and counted by scintillation as described below. A 2-mL sample was pipetted from the $\mathrm{S} 4$ and then analyzed as for the other fractions.

To ascertain the composition of the subcellular fractions, nonradioactive brains were included in some runs, and the fractions were examined with electron microscopy (23).

Analytical methods. Serum bilirubin was measured by a diazo method, and hematocrit was measured with microhematocrit tubes. The protein content of the subcellular fractions was measured with the BioRad DC Protein Assay (Bio-Rad Laboratories, Hercules, CA, U.S.A.). Serum osmolality was measured with a vapor pressure osmometer.

Subcellular fractions $(1.8 \mathrm{~mL})$ were mixed with $10 \mathrm{~mL}$ Ultima Gold scintillation fluid and counted for $1 \mathrm{~min}$ in a Wallac 1410 Liquid Scintillation Counter (Wallac Inc., Gaithersburg, MD, U.S.A.). Duplicate $10-\mu \mathrm{L}$ serum samples obtained at the time of sacrifice were counted in the same way, and the specific radioactivity for bilirubin at the time of sacrifice was calculated from the serum bilirubin value at sacrifice.

Statistical methods. The bilirubin concentrations in the various subcellular compartments were calculated in relation to protein content, and were contrasted with ANOVA. Because of heterogeneity of variances, the Kruskal-Wallis nonparametric ANOVA was used, followed by Dunn's multiple comparisons test. The level of significance was taken as $p<0.05$.

\section{RESULTS}

Serum bilirubin values (mean $\pm \mathrm{SD}$ ) for rats killed at $10 \mathrm{~min}$ were $529 \pm 78 \mu \mathrm{M}$ (controls) versus $497 \pm 74 \mu \mathrm{M}$ (hypercarbia) versus $540 \pm 178 \mu \mathrm{M}$ (hyperosmolality) with no statistically significant difference among the groups. Similarly there were no significant intergroup differences in serum bilirubin values for rats killed at $30 \min (394 \pm 42 \mu \mathrm{M}$ versus $359 \pm 40 \mu \mathrm{M}$ versus $463 \pm 126 \mu \mathrm{M})$. As expected, serum bilirubin decreased significantly between the two time points ( $p$ $=0.0005)$. Hematocrit was approximately $40 \%$ in all groups at time 0 , and because of sampling-related losses fell to approximately $30 \%$ at the time of sacrifice (no intergroup differences).

As intended, serum osmolality was significantly elevated in the rats that had received urea $(408 \pm 31 \mathrm{mosm} / \mathrm{L})$. From previous experience, serum osmolality is typically $285-300$ mosm $/ \mathrm{L}$ in rats and does not change in control and hypercarbic rats under these experimental conditions (22). It was therefore only checked for control purposes in four control rats and was found to be $288 \pm 16 \mathrm{mosm} / \mathrm{L}$. In the rats that breathed $\mathrm{CO}_{2}$, $\mathrm{pH}$ fell to $6.89 \pm 0.07$, and $\mathrm{Pco}_{2}$ increased to $14.49 \pm 5.83$ $\mathrm{kPa}$.
Differences in bilirubin concentration as related to protein content among rat brain subcellular compartments were highly significant in all groups and at all time points (Fig. 1).

\section{DISCUSSION}

This is the first study to address the question of bilirubin subcellular localization in brain. Our results show that bilirubin appears to be more concentrated in some subcellular compartments than in others. The profile of bilirubin subcellular distribution is modulated by the conditions under which bilirubin enters the brain.

Under all conditions there were relatively high concentrations of bilirubin in the myelin fraction. This observation is interesting in light of theories and data suggesting that membranes are the primary target of bilirubin toxicity support (4-10). Myelin contains approximately $30 \%$ protein and $70 \%$ lipid, of which nearly half is cholesterol. Whether the lipophilicity of bilirubin plays any role in this context cannot be ascertained by the present data. The very high concentration of bilirubin relative to protein in cytoplasm, ribosomes, and mitochondria in the hyperosmolar group are also notable in light of data from hyperbilirubinemic animals in which changes in electrophysiology or energy metabolism only appeared after hyperosmolar opening of the blood-brain barrier (25). It is also noteworthy that data from a study on short-term bilirubin entry into whole brain did not show an increase during hyperosmolality relative to control conditions, although bilirubin clearance from brain was significantly delayed (22). In the present study, bilirubin concentrations in subcellular compartments were higher in animals exposed to hyperosmolality than in control or hypercarbic animals. This suggests the possibility that hyperosmolar opening of the blood-brain barrier may facilitate the entry of bilirubin into brain cells. The persistently high bilirubin concentration in cytoplasm at $30 \mathrm{~min}$ is compatible with the previous finding of delayed bilirubin clearance from whole brain (22).

Data from whole brain have shown that hypercarbia is associated with a more rapid entry of bilirubin into brain in high concentrations than under control conditions (22). The present data do not show a similar phenomenon as far as the subcellular fractions. Increased bilirubin entry into brain in hypercarbia has been thought to be related to increased brain blood flow (26), although some opening of the blood-brain barrier probably also occurs (27). The reason for the apparent difference in the kinetics of bilirubin entry into whole brain versus subcellular fractions during respiratory acidosis is not intuitively obvious. This phenomenon may require further study.

The designation of the $\mathrm{P} 1$ fraction as nuclei and the $\mathrm{S} 4$ as cytoplasm is an approximation. Although the P1 contents are to a large extent nuclei, tissue fragments and portions of blood vessels are also found in this fraction (23). The contents of the S4 are not well characterized, but may contain some ribosomes, although the electron micrographs of our S4 fractions did not contain any such structures. The protein content is very low, so that the apparently high concentration of bilirubin in this fraction, particularly from the hyperosmolar animals, may 


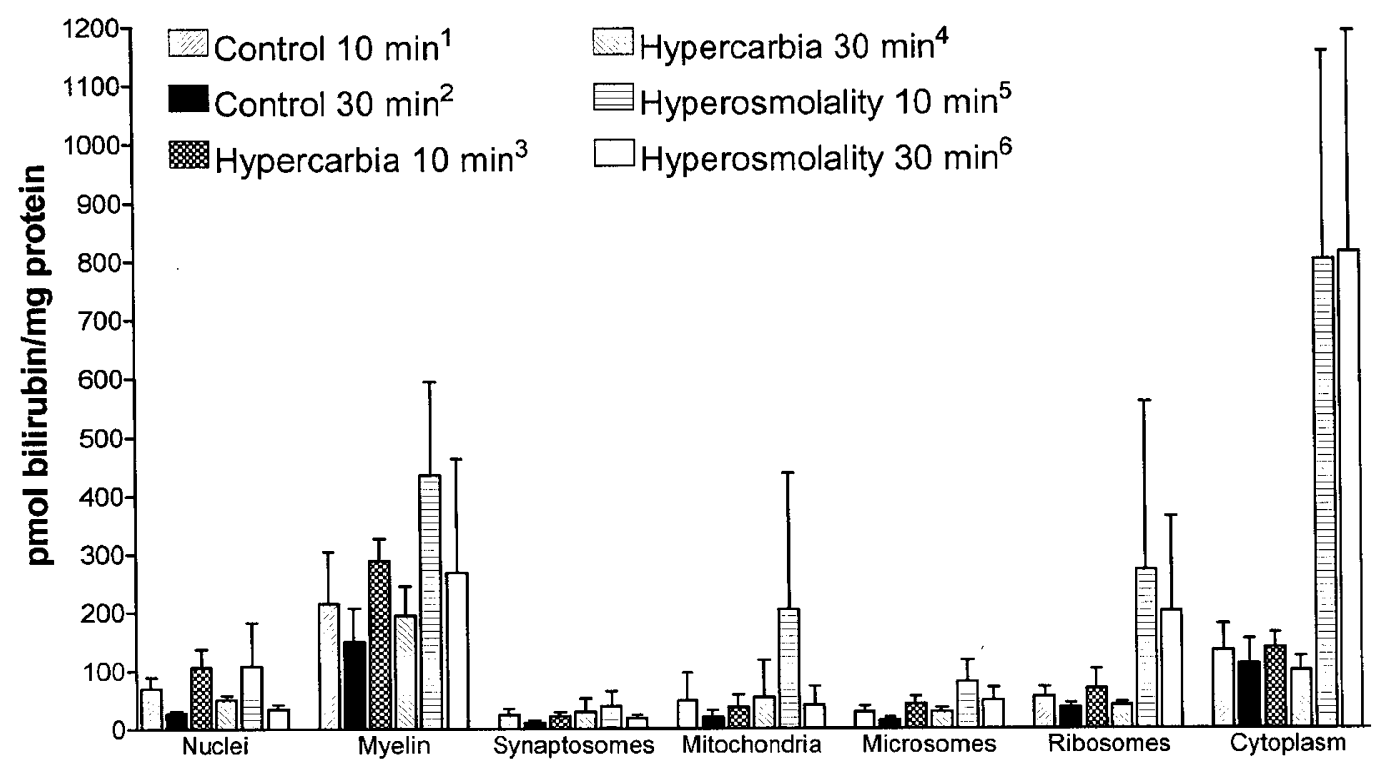

Fig. 1. Bilirubin concentration (pmol bilirubin per mg protein; mean $\pm \mathrm{SD}$ ) in rat brain subcellular organelles according to group (control, hypercarbia, hyperosmolality) and time of sacrifice after an i.v. bilirubin bolus (10 min $v s 30 \mathrm{~min}$ ). ${ }^{1}$ Kruskal-Wallis statistic $=35.8 ; p<0.0001$. Significant contrasts using Dunn's multiple comparisons test: myelin $v s$ microsomes and synaptosomes, $p<0.001$; myelin $v s$ mitochondria, $p<0.01 .{ }^{2}$ Kruskal-Wallis statistic $=43.2$; $p<0.0001$. Dunn's multiple comparisons test: myelin $v s$ microsomes and synaptosomes, $p<0.001$; myelin $v s$ mitochondria, $p<0.01$; cytoplasm $v s$ synaptosomes and microsomes, $p<0.001$; cytoplasm $v$ s mitochondria, $p<0.05$. ${ }^{3}$ Kruskal-Wallis statistic $=40.0 ; p<0.0001$. Dunn's multiple comparisons test: myelin $v s$ synaptosomes and mitochondria, $p<0.001$; myelin $v s$ microsomes, $p<0.01$; cytoplasm $v s$ synaptosomes and mitochondria, $p<0.01$. ${ }^{4}$ Kruskal-Wallis statistic $=32.2 ; p<0.0001$. Dunn's multiple comparisons test: myelin $v s$ microsomes and synaptosomes, $p<0.001$; myelin $v s$ mitochondria and cytoplasm, $p<0.05$; cytoplasm $v s$ synaptosomes and microsomes, $p<0.05$. ${ }^{5}$ Kruskal-Wallis statistic $=27.3 ; p=0.0001$. Dunn's multiple comparisons test: myelin $v$ synaptosomes, $p<0.01$; cytoplasm $v$ s synaptosomes, $p<0.001$; cytoplasm $v s$ microsomes, $p<0.05$. ${ }^{6}$ Kruskal-Wallis statistic $=36.5$; $p<$ 0.0001. Dunn's multiple comparisons test: nuclei $v s$ cytoplasm, $p<0.01$; myelin $v s$ synaptosomes, $p<0.01$; synaptosomes $v s$ ribosomes, $p<0.05$; synaptosomes $v$ cytoplasm, $p<0.001$; mitochondria $v s$ cytoplasm, $p<0.01$; microsomes $v s$ cytoplasm, $p<0.05$.

perhaps in part be an artifact. However, it is noteworthy that brain glutathione $S$-transferase is especially abundant in the cytosol fraction (28). This brain enzyme was shown to bind bilirubin, but less strongly than the liver enzyme (ligandin) and albumin.

There are many theories concerning the basic mechanism for bilirubin neurotoxicity. All in vitro studies concerning bilirubin effects on cellular functions must be interpreted in light of the question of whether the concentrations of bilirubin used are physiologically relevant. The concentrations found in subcellular compartments in the present study seem to suggest that many previous in vitro studies may have been performed under conditions in which bilirubin concentrations relative to protein were supraphysiologic.

However, caution is needed in interpreting the present data. During fractionation all subcellular fractions are diluted in a watery phase, and their final concentration as well as localization in the gradient will be related to the specific gravity of the particle and the density of the solution in which it is suspended. Measuring absolute bilirubin concentrations in such phases would therefore not have provided meaningful information regarding intracellular conditions. Therefore, bilirubin concentrations had to be measured relative to some other cellular constituent. As bilirubin is known to bind to proteins (e.g. ligandin and albumin), relating bilirubin content to protein seemed a reasonable approximation. As mentioned briefly above, bilirubin binds to brain glutathione $S$-transferase, which is enriched in the cytosol, and to some extent in microsomes and mitochondria (28). Cytosolic glutathione $S$-transferases in rat brain are increased in hyperbilirubinemia, and the regional pattern of distribution is altered (29). Battiston et al. (30) recently detected two peptides in rat brain (79 and $34 \mathrm{kD})$ that bound bilirubin. However, nothing is known about the potential binding of bilirubin to other specific brain proteins. In the present study only the content of bilirubin relative to total protein concentration was calculated. When and if information on bilirubin binding to other specific brain protein(s) becomes available, it may be desirable to compare the bilirubin content of subcellular fractions to the concentration of specific binding protein(s).

Inasmuch as bilirubin is a lipophilic compound, it may also be relevant to relate bilirubin concentrations to the lipid content of the subcellular fractions. We analyzed lipid content (as cholesterol) in the subcellular fractions in control animals, and found this to be very low. As cholesterol constitutes nearly half of membrane lipids, attempting to relate bilirubin content to other lipids did not seem more likely to be helpful. For this reason we are not convinced that these data are reliable, and have elected not to present the data. However, it appeared that the distribution profile of bilirubin across subcellular fractions when related to cholesterol was similar to that seen in relation to protein.

We have only studied the subcellular localization of bilirubin in brain during short-term hyperbilirubinemia. It is possible that bilirubin in brain cells during longer-lasting hyperbilirubinemia may distribute differently. We elected to use a radio- 
active label to measure bilirubin content in subcellular fractions, as it was not clear that alternative analytical methods with adequate sensitivity were available. Bilirubin is metabolized in brain (31-35). Therefore it would have been difficult to be certain that the label still represented (only) bilirubin during prolonged hyperbilirubinemia. Thus, the answer to the question of bilirubin subcellular localization in brain during prolonged hyperbilirubinemia will probably require a different approach.

In the present study bilirubin was found to distribute differentially in brain subcellular compartments. It was found to be highly associated with the myelin (i.e. membrane) fraction in all three experimental conditions (control, respiratory acidosis, and hyperosmolality). The high concentration of bilirubin found in the membrane fraction does not prove that the primary mechanism of bilirubin neurotoxicity is necessarily associated with this fraction. During hyperosmolality elevated concentrations of bilirubin were also found in mitochondria, ribosomes, and final supernatant (cytoplasm), a pattern of distribution that has some similarity to brain glutathione $S$-transferase (28).

The search for the basic mechanism for bilirubin neurotoxicity continues. Knowing the concentrations of bilirubin associated with different subcellular compartments may be of value in designing and evaluating studies of the effects of bilirubin on basic cellular functions.

Acknowledgment. The authors thank Kelly Ann Randall for preparing the electron micrographs.

\section{REFERENCES}

1. Vaetterli II 1753 Dissertatio inauguralis medica de ictero illoque speciatim, quo infantes recens nati laborant. I.C.L.Schulz, Göttingen, pp $1-25$

2. Zetterström R, Ernster L 1956 Bilirubin, an uncoupler of oxidative phosphorylation in isolated mitochondria. Nature 178:1335-1337

3. Day RL 1954 Inhibition of brain respiration in vitro by bilirubin: reversal of inhibition by various means. Am J Dis Child 88:504-506

4. Cowger ML 1971 Mechanism of bilirubin toxicity on tissue culture cells: factors that affect toxicity, reversibility by albumin, and comparison with other respiratory poisons and surfactants. Biochem Med 5:1-16

5. Hackney DD 1980 Photodynamic action of bilirubin on the inner mitochondrial membrane: implications for the organization of the mitochondrial ATPase. Biochem Biophys Res Commun 94:875-880

6. Kaul R, Bajpai VK, Shipstone AC, Kaul HK, Murti CRK 1981 Bilirubin-induced erythrocyte membrane cytotoxicity. Exp Mol Pathol 34:290-298

7. O'Callaghan A, Duggan PF 1984 Possible biochemical basis for bilirubin neurotoxicity. Biochem Soc Trans 12:483-483

8. Ochoa EL, Wennberg RP, An Y, Tandon T, Takashima T, Nguyen T, Chui A 1993 Interactions of bilirubin with isolated presynaptic nerve terminals: functional effects on the uptake and release of neurotransmitters. Cell Molec Neurobiol 13:69-86

9. Vazquez J, Garcia-Calvo M, Valdivieso F, Mayor F, Mayor Jr F 1988 Interaction of bilirubin with the synaptosomal plasma membrane. J Biol Chem 263:1255-1265
10. Hansen TWR, Paulsen O, Gjerstad L, Bratlid D 1988 Short-term exposure to bilirubin reduces synaptic activation in rat transverse hippocampal slices. Pediatr Res 23:453456

11. Hansen TWR, Bratlid D 1986 Bilirubin and brain toxicity. Acta Paediatr Scand 75:513-522

12. Morphis L, Constantopoulos A, Matsaniotis N 1982 Bilirubin-induced modulation of cerebral protein phosphorylation in neonate rabbits in vivo. Science 218:156-158

13. Constantopoulos A, Matsaniotis N 1976 Bilirubin inhibition of protein kinase: its prevention by cyclic AMP. Cytobios 17:17-20

14. Hansen TWR, Bratlid D, Walaas SI 1988 Bilirubin decreases phosphorylation of synapsin I, a synaptic vesicle-associated neuronal phosphoprotein, in intact synaptosomes from rat cerebral cortex. Pediatr Res 23:219-223

15. Hansen TWR, Mathiesen SBW, Walaas SI 1996 Bilirubin has widespread inhibitory effects on protein phosphorylation. Pediatr Res 39:1072-1077

16. Hansen TWR, Mathiesen SBW, Walaas SI 1997 Modulation of the effect of bilirubin on protein phosphorylation by lysine-containing peptides. Pediatr Res 42:615-617

17. Rapoport SI 1983 Reversible osmotic opening of the blood-brain barrier for experimental and therapeutic purposes. In: Levine RL, Maisels MJ (eds) Hyperbilirubinemia in the Newborn. Report of the 85th Ross Conference on Pediatric Research, Ross Laboratories, Columbus, OH, pp 116-120

18. Bratlid D 1984 Mechanism of bilirubin entry into the brain in an animal model. In: Rubaltelli F, Jori J (eds) Neonatal Jaundice: New Trends in Phototherapy. Plenum Press, New York, pp 23-34

19. Levine RL, Fredericks WR, Rapoport SI 1982 Entry of bilirubin into the brain due to opening of the blood- brain barrier. Pediatrics 69:255-259

20. Hansen TWR 1995 Acute entry of bilirubin into rat brain regions. Biol Neonate 67:203-207

21. Hansen TWR, Cashore WJ 1995 Rates of bilirubin clearance from rat brain regions Biol Neonate 68:135-140

22. Hansen TWR 1996 Bilirubin entry into and clearance from rat brain during hypercarbia and hyperosmolality. Pediatr Res 39:72-76

23. Gray EG, Whittaker VP 1962 The isolation of nerve endings from brain: an electron-microscopic study of cell fragments derived by homogenization and centrifugation. J Anat 96:79-88

24. Whittaker VP, Barker LA 1972 The subcellular fractionation of brain tissue with special reference to the preparation of synaptosomes and their component organelles. In: Fried R (ed) Methods of Neurochemistry, Vol.2. Marcel Dekker, New York, pp $1-52$

25. Wennberg RP, Johansson BB, Folbergrova J, Siesjö BK 1991 Bilirubin-induced changes in brain energy metabolism after osmotic opening of the blood-brain barrier. Pediatr Res 30:473-478

26. Robinson PJ, Rapoport SI 1987 Binding effect of albumin on uptake of bilirubin by brain. Pediatrics 79:553-558

27. Hansen TWR, Øyasæter S, Stiris T, Bratlid D 1989 Effects of sulfisoxazole, hypercarbia, and hyperosmolality on entry of bilirubin and albumin into brain regions in young rats. Biol Neonate 56:22-30

28. Asaoka K, Takahashi K 1983 Purification and properties of porcine brain glutathione $S$-transferases. J Biochem 94:1191-1199

29. Johnson JA, Hayward JJ, Kornguth SE, Siegel FL 1993 Effects of hyperbilirubinaemia on glutathione $S$-transferase isoenzymes in cerebellar cortex of the Gunn rat. Biochem J 291:453-461

30. Battiston L, Macagno A, Passamonti S, Micali F, Sottocasa GL 1999 Specific sequence-directed anti-bilitranslocase antibodies as a tool to detect potentially bilirubin-binding proteins in different tissues of the rat. FEBS Lett 453:351-355

31. Hansen TWR, Allen J 1996 Bilirubin-oxidizing activity in rat brain. Biol Neonate 70:289-295

32. Hansen TWR, Allen JW 1997 Oxidation of bilirubin by brain mitochondrial membranes-dependence on cell type and postnatal age. Biochem Molec Med 60:155160

33. Hansen TWR, Tommarello S, Allen JW 1997 Oxidation of bilirubin by rat brain mitochondrial membranes - genetic variability. Biochem Molec Med 62:128-131

34. Hansen TWR, Tommarello S 1998 Effects of phenobarbital on bilirubin clearance and metabolism in rat brain. Biol Neonate 73:106-111

35. Hansen TWR, Allen JW, Tommarello S 1999 Oxidation of bilirubin in the brainfurther characterization of a potentially protective mechanism. Molec Genet Metab 68:404-409 